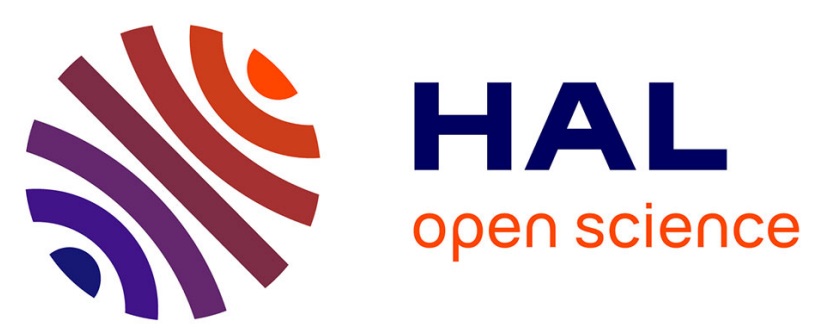

\title{
A fast method for the quantification of fat fraction and relaxation times: Comparison of five sites of bone marrow.
}

Caroline Le Ster, Jérémy Lasbleiz, Stephan Kannengiesser, Raphaël Guillin, Giulio Gambarota, Hervé Saint-Jalmes

\section{To cite this version:}

Caroline Le Ster, Jérémy Lasbleiz, Stephan Kannengiesser, Raphaël Guillin, Giulio Gambarota, et al.. A fast method for the quantification of fat fraction and relaxation times: Comparison of five sites of bone marrow.. Magnetic Resonance Imaging, 2017, 39, pp.157-161. 10.1016/j.mri.2017.03.001 . hal-01508422

\section{HAL Id: hal-01508422}

\section{https://hal-univ-rennes1.archives-ouvertes.fr/hal-01508422}

Submitted on 7 Jul 2017

HAL is a multi-disciplinary open access archive for the deposit and dissemination of scientific research documents, whether they are published or not. The documents may come from teaching and research institutions in France or abroad, or from public or private research centers.
L'archive ouverte pluridisciplinaire HAL, est destinée au dépôt et à la diffusion de documents scientifiques de niveau recherche, publiés ou non, émanant des établissements d'enseignement et de recherche français ou étrangers, des laboratoires publics ou privés. 
A fast method for the quantification of fat fraction and relaxation times: comparison of five sites of bone marrow.

Caroline Le Ster ${ }^{a, b, c}$, Jérémy Lasbleiz ${ }^{a, b}$, Stephan Kannengiesser ${ }^{d}$, Raphaël Guillin ${ }^{\mathrm{e}}$, Giulio Gambarota $^{\mathrm{a}, \mathrm{b}}$ and Hervé Saint-Jalmes $\mathrm{s}^{\mathrm{a}, \mathrm{b}, \mathrm{f}}$

a INSERM, UMR 1099, Rennes, France

${ }^{\mathrm{b}}$ LTSI, Université de Rennes 1, Rennes, France

${ }^{\mathrm{c}}$ Siemens Healthcare, Saint-Denis, France

${ }^{\mathrm{d}}$ Siemens Healthcare, Erlangen, Germany

e Department of imaging, Rennes University Hospital, Rennes, France

${ }^{f} \mathrm{CRLCC}$, Centre Eugène Marquis, Rennes, France

E-mail addresses: le.ster.caro@gmail.com, jeremy.lasbleiz@gmail.com, stephan.kannengiesser@siemens.com, raphael.guillin@chu-rennes.fr, gambarota@gmail.com, herve.saint-jalmes@univ-rennes1.fr

Corresponding author: Caroline Le Ster, INSERM U1099, LTSI Université de Rennes 1, 35000 Rennes, France, +33223234849, le.ster.caro@gmail.com 


\section{Abstract \\ Purpose}

Bone marrow is found either as red bone marrow, which mainly contains haematopoietic cells, or yellow bone marrow, which mainly contains adipocytes. In adults, red bone marrow is principally located in the axial skeleton. A recent study has introduced a method to simultaneously estimate the fat fraction $(\mathrm{FF})$, the $\mathrm{T} 1$ and $\mathrm{T} 2^{*}$ relaxation times of water $(\mathrm{T} 1 \mathrm{w}$, $\left.T 2^{*} w\right)$ and fat $\left(T 1 f\right.$ and $\left.T 2^{*} f\right)$ in the vertebral bone marrow. The aim of the current study was to measure FF, T1w, T1f, T2* $\mathrm{w}$ and $\mathrm{T} 2^{*} \mathrm{f}$ in five sites of bone marrow, and to assess the presence of regional variations.

\section{Methods}

MRI experiments were performed at $1.5 \mathrm{~T}$ on five healthy volunteers $(31.6 \pm 15.6$ years $)$ using a prototype chemical-shift-encoded 3D multi-gradient-echo sequence (VIBE) acquired with two flip angles. Acquisitions were performed in the shoulders, lumbar spine and pelvis, with acquisition times of less than 25 seconds per sequence. Signal intensities of magnitude images of the individual echoes were used to fit the signal and compute $F F, T 1 w, T 1 f, T 2{ }^{*} w$ and $\mathrm{T}^{*} \mathrm{f}$ in the humerus, sternum, vertebra, ilium and femur.

\section{Results}

Regional variations of fat fraction and relaxation times were observed in these sites, with higher fat fraction and longer T1w in the epiphyses of long bones. A high correlation between FF and T1w was measured in these bones $(R=0.84$ in the humerus and $R=0.84$ in the femur). In most sites, there was a significant difference between water and fat relaxation times, attesting the relevance of measuring these parameters separately.

\section{Conclusion}

The method proposed in the current study allowed for measurements of $F F, T 1 w, T 1 f, T 2{ }^{*} w$ and T2* $\mathrm{f}$ in five sites of bone marrow. Regional variations of these parameters were observed and a strong negative correlation between the T1 of water and the fat fraction in bones with high fat fractions was found. 


\section{Keywords}

Water-fat imaging; Relaxometry; Bone Marrow.

List of abbreviations used: VIBE, volumetric interpolated breath-hold examination;

CAIPIRINHA, controlled aliasing in parallel imaging results in higher acceleration; CV, coefficient of variation. 


\section{Introduction}

Bone marrow is a tissue located in the central cavities of the long bones and in the cavities of the trabecular bones. This tissue is principally composed of haematopoietic cells and adipocytes, and it is responsible for the production of erythrocytes, platelets and leucocytes

$[1,2]$. Bone marrow is found either as red bone marrow, which mainly contains haematopoietic cells or yellow bone marrow, which mainly contains adipocytes. Red bone marrow is predominant during childhood; it is then gradually replaced by yellow bone marrow. In adults, red bone marrow principally remains in the axial skeleton, namely the skull, ribs, sternum, scapulae and vertebrae, as well as in parts of the peripheral skeleton: in the ilium and the epiphyses of the humerus and the femur [1].

Quantitative chemical-shift-encoded MRI has been widely used to study bone marrow composition. A recent study [3] has shown the presence of regional variations in the composition of bone marrow with higher fat fraction and lower diffusion coefficient in the peripheral skeleton compared to the axial skeleton. Quantitative MRI can also be used as a non-invasive tool to diagnose bone marrow pathologies. The fat fraction and the T1 and T2* relaxation times have been shown to be relevant biomarkers of the pathophysiological status of this tissue. For instance, the vertebral fat fraction decreased in patients suffering from bone marrow malignancies [4] and it increased after treatment in patients who received chemotherapy [5]; the vertebral fat fraction was found to be higher in osteoporotic patients than in healthy volunteers $[6,7]$. The quantification of $\mathrm{T} 1$ and $\mathrm{T} 2^{*}$ is also of interest in several pathologies of the bone marrow. For instance, in a number of studies it was found that osteoporotic patients had a longer vertebral common-T2* $[8-11]$ and a shorter vertebral common-T1 [11] than healthy volunteers; and that the T1 of water was higher in vertebrae with malignancies than in healthy vertebrae $[12,13]$.

Recently, a method based on fast chemical-shift imaging was proposed to measure the fat fraction and the separate $T 1$ and $T 2^{*}$ of water and fat $\left(F F, T 1 w, T 1 f, T 2^{*} w\right.$ and $\left.T 2^{*} f\right)$ in the bone marrow with two acquisitions [14]. In this method, the MR signal is modelled as the 
weighted sum of water and fat signals and the signal intensity sampled in the tissue is fitted to the signal equation to quantify FF, T1w, T1f, T2* $w$ and $T 2^{*} f$.

As previous studies have shown regional variations of bone marrow fat fractions, and given the importance of $\mathrm{T} 1$ and $\mathrm{T} 2^{*}$ as biomarkers, the aim of the current study was to measure FF, T1w, T1f, T2* $w$ and T2* in multiple sites of bone marrow in healthy volunteers and to assess the presence of regional variations for these parameters.

\section{Materials and methods}

\subsection{Magnetic Resonance Imaging (MRI)}

All experiments were conducted according to the procedures approved by the local Institutional Review Board. A group of 5 healthy volunteers (age range 20-58 years, mean age $31.6 \pm 15.6$ years, 3 women and 2 men) were enrolled in the current study. Experiments were performed on a 1.5T MR system (MAGNETOM Aera, Siemens Healthcare, Erlangen, Germany) in the shoulders (coronal acquisition), the lumbar spine (sagittal acquisition) and the pelvis (coronal acquisition) using the regular spine and body receiver coils. The imaging protocol consisted of a prototype chemical-shift-encoded 3D multi-gradient-echo sequence (volumetric interpolated breath-hold examination, VIBE [15]) acquired with two flip angles in consecutive runs.

The scanning parameters used in the VIBE sequence were optimised in a previous study [14] and were as follows: repetition time $T R=8.21 \mathrm{~ms}$, four echoes acquired in a single TR (TE1/TE2/TE3/TE4 = 1.18/2.34/4.4/6.8 ms), bandwidth $1220 \mathrm{~Hz} /$ pixel, parallel imaging using CAIPIRINHA (controlled aliasing in volumetric parallel imaging results in higher acceleration) with an acceleration factor $\mathrm{R}=2$ in the phase encoding direction and $\mathrm{R}=2$ in the slice encoding direction resulting in a total acceleration factor of $R=4,60$ slices of $4 \mathrm{~mm}$ thickness; the sequence was performed with flip angles of $5^{\circ}$ and $15^{\circ}$. Sagittal acquisitions in the lumbar spine were performed with: matrix $192 \times 256$, field of view $315 \times 420 \mathrm{~mm}^{2}$ and partial Fourier 6/8, resulting in an acquisition time of 16 seconds per sequence. Coronal acquisitions in the shoulder and the pelvis were performed with: matrix $256 \times 256$, field of view 
$420 \times 420 \mathrm{~mm}^{2}$ and no partial Fourier, resulting in an acquisition time of 23 seconds per sequence. Lumbar spine and shoulder acquisitions were performed with breath-holds.

\subsection{Data analysis}

Regions of interest (ROIs) were drawn with ImageJ (NIH, Bethesda, MD, http://imagej.nih.gov/ij/) by a radiologist (J.L., 15 years of experience). Twelve ROls were drawn for each volunteer: one in each the upper epiphyses of the right and left humerus, one in the sternum, one in each of the five lumbar vertebrae L1 to L5 (in the anterior part of the vertebral body to avoid vascular artifacts), one in each lateral side of the ilium and one in each the upper epiphyses of the right and left femur. The ROls were placed on the images acquired at TE2 with the flip angle of $5^{\circ}$, and were copied to the other images. Each ROI had an area of 52 voxels and was positioned in the bone marrow with care to avoid voxels with cortical bone and partial volume effects. For each of the twelve ROls, ROI-averaged signal intensities and standard deviations were measured in the magnitude images of the four individual echoes acquired with the two flip angles.

Signal analysis was performed according to the method described in [14]. In this method, the signal intensity of a spoiled gradient echo sequence is modelled as the weighted sum of water and fat signals with separate T1 and T2* relaxation times for water and fat. In contrast to the method described in [14], the fat signal model used here consisted of the four main peaks of the vertebral bone marrow MR spectrum $(0.9,1.3,2-2.2$ and $5.3 \mathrm{ppm}$ with relative peak areas of $9 \%, 63 \%, 15 \%$ and $7 \%$, respectively) recently characterised by Karampinos et al. [16]. The signal intensity is therefore a function of the sequence parameters (TR, TE and flip angle) and the tissue parameters (S0, FF, T1w, T1f, T2* $w$ and T2*f). In each of the twelve ROIs, the averaged signal measured on the magnitude images acquired at the four echo times and with the two flip angles was fitted to the signal equation, and FF, T1w, T1f, $T 2^{*} \mathrm{~W}$ and $\mathrm{T} 2^{*} \mathrm{f}$ were determined. The ambiguity between water and fat signals arising from the use of magnitude data was resolved by assuming that T1w was higher than T1f 
$[17,18]$.The fitting was performed with in-house-built scripts written in Mathematica (Wolfram Research, Champaign, IL, USA).

The parameters computed in the right and left humerus were averaged, and so were the parameters computed in the five lumbar vertebrae (L1 to L5), in the right and left ilium and in the right and left femur.

\subsection{Statistical analysis}

The coefficient of variation (CV) was computed for each parameter (FF, T1w, T1f, T2* $\mathrm{w}$ and T2*f) in the five sites of bone marrow studied. The differences between the cohort-mean water and fat relaxation times were assessed with t-tests for $\mathrm{T} 1$ relaxation times ( $\mathrm{T} 1 \mathrm{w}$ versus T1f) and for $T 2^{*}$ relaxation times $\left(T 2^{*} w\right.$ versus $\left.T 2^{*} f\right)$ on each site. The presence of a correlation between the fat fraction and the T1 of water was assessed by computing the Pearson correlation coefficient for each site. The threshold $p<0.01$ was chosen for statistical significance. Statistical analyses were performed with MATLAB (MathWorks, Natick, MA, USA).

\section{Results}

A set of MR images acquired on a volunteer at the first echo time (TE=1.18 $\mathrm{ms}$ ) is displayed in Figure 1; it represents the five sites of bone marrow studied. Data fitting and parameter quantification were successful for all sites despite a wide range of tissue parameters encountered; the example of a signal fit performed in one vertebra and in the humerus is shown in Figure 2 . The averaged values for fat fraction, $\mathrm{T} 1$ and $\mathrm{T} 2^{*}$ relaxation times of water and fat computed in the bone marrow of the humerus, sternum, vertebra, ilium and femur are given in Table 1. Several results from this table can be outlined. First, the fat fractions were higher in the epiphyses of long bones (humerus and femur) than in the sternum, vertebra and ilium; for instance the mean fat fraction was $74 \pm 11 \%$ in the humerus versus $42 \pm 15 \%$ in the vertebra. In addition, the inter-individual variability of the fat fraction depended on the site of bone marrow considered: the coefficient of variation ranged between $8 \%$ in the ilium and 
$32 \%$ in the sternum. Second, the estimates of T1 $\mathrm{w}$ varied from $621 \pm 110 \mathrm{~ms}$ in the vertebra to $815 \pm 333 \mathrm{~ms}$ in the humerus with a large inter-individual variability (CVs between $18 \%$ in the vertebra and $54 \%$ in the femur); the estimates of T1f varied from $224 \pm 92 \mathrm{~ms}$ in the sternum to $330 \pm 20 \mathrm{~ms}$ in the vertebra with a smaller inter-individual variability (lower CVs). Third, the estimates of $T 2^{*} \mathrm{w}$ varied from $4.7 \pm 3.5 \mathrm{~ms}$ in the humerus to $12.7 \pm 5.4 \mathrm{~ms}$ in the vertebra. The estimates of T2f varied from $15.9 \pm 3.9 \mathrm{~ms}$ in the vertebra to $30.0 \pm 2.4 \mathrm{~ms}$ in the humerus. The coefficients of determination of the fitting curve were close to 1 for each site of bone marrow $\left(R^{2}>0.999\right)$. Results of the t-test are given in Table 2 . Differences between the mean T1 of water and the mean T1 of fat were significant in all the sites of bone marrow studied ( $p<0.01$ ) with T1w longer than T1f (as was initially assumed). Differences between the $\mathrm{T}^{*}$ of water and the $\mathrm{T} 2^{*}$ of fat were not significant in the vertebrae, whereas they were significant in other sites, with $T 2^{*}$ f longer than $T 2^{*} \mathrm{~W}$.

Pearson correlation coefficients computed between the fat fraction and the T1 of water were: -0.84 in the humerus $(p=0.003),-0.70$ in the sternum $(p=0.19),-0.32$ in the vertebrae $(p=0.11), 0.59$ in the ilium $(p=0.07)$ and -0.84 in the femur $(p=0.003)$. Thus there was a strong negative correlation between the fat fraction and the $\mathrm{T} 1$ of water in bones with high fat fraction (Figure 3), namely the epiphyses of the humerus and the femur, whereas no significant correlation was measured in other sites $(p>0.01)$.

\section{Discussion}

In the current study we determined the fat fraction, $\mathrm{T} 1$ and $\mathrm{T} 2^{*}$ relaxation times of water and fat in five sites of bone marrow in healthy volunteers using a fast chemical-shift-encoded 3D multi-gradient-echo sequence. In a previous study, Schraml et al. reported regional variations of bone marrow fat fractions using the two-point Dixon method [3]. In the current study, the fat fraction, $\mathrm{T} 1$ and $\mathrm{T} 2^{*}$ relaxation times of water and fat were simultaneously computed to allow a further insight into the composition of bone marrow across locations. The repeatability of the method employed in the current study was assessed in a previous work on the vertebral bone marrow [14] by scanning one volunteer six times. The mean 
coefficients of variation obtained from this repeatability study were: $8 \%(\mathrm{FF}), 11 \%(\mathrm{~T} 1 \mathrm{w})$, $17 \%(\mathrm{~T} 1 \mathrm{f}), 8 \%\left(\mathrm{~T} 2^{*} \mathrm{~W}\right)$ and $27 \%\left(\mathrm{~T} 2^{*} \mathrm{f}\right)$. In the literature, the fat fraction of the vertebral bone marrow has been shown to vary with age and sex [19-23], with an increase of the fat fraction with age and higher fat fractions in men than in women. Kugel et al. [19] measured a mean fat fraction of $37 \pm 11 \%$ in L3 with spectroscopy in a population of $31-40$ years of age (matched to our own population) and more recently, Baum et al. [24] measured a mean fat fraction of $36 \pm 10 \%$ in L1-L5 in a population of $26 \pm 4$ years of age of healthy volunteers. $A$ wide range of relaxation times were measured in the lumbar vertebrae for the T1 of water: at 1.5T, the T1w was $872 \pm 129 \mathrm{~ms}$ in the study of Biffar et al. [12] (imaging), $901 \pm 13 \mathrm{~ms}$ in the study of Träber et al. [13] (spectroscopy) and 1000-1700 ms in the study of Schick et al. [25] (spectroscopy). The T1 of fat measured in the lumbar vertebrae was $324 \pm 81 \mathrm{~ms}$ in the study of Biffar et al., $266 \pm 2 \mathrm{~ms}$ in the study of Träber et al. and $260-320 \mathrm{~ms}$ in the study of Schick et al. A common-T2* was measured by Funke et al. [9] and Wehrli et al. [10] in the vertebrae of healthy volunteers at $1.5 \mathrm{~T}$, the values ranged between $13.4 \mathrm{~ms}$ and $15.4 \mathrm{~ms}$, respectively. The vertebral T1 of water measured in the current study was slightly lower than the values reported in the literature. Overall, results obtained in the current study for the vertebral bone marrow are in agreement with the literature.

Recent studies have shown a large heterogeneity in bone marrow fat fractions across sites: Schraml et al. [3] used the two-point Dixon method to measure the fat fraction in the bone marrow of the humerus (93 $\pm 8 \%)$, lumbar vertebrae $(62 \pm 16 \%)$, pelvis $(74 \pm 13 \%)$ and femur $(89 \pm 7 \%)$ in a population of $54 \pm 19$ years of age suffering from different types of cancer. Bolan et al. [5] used the three-point Dixon method [26] to measure the fat fraction in the bone marrow of the L4 lumbar vertebra (median of $47 \%$ ) and femur (median of $78 \%$ ) in a population of 35-63 years of age suffering from gynaecological malignancies (pre-treatment fat fractions). The fat fractions measured in these studies showed variations similar to the current study: higher fat fractions in the peripheral skeleton compared to the axial skeleton. This result can be explained by the centripetal conversion of the bone marrow [27]: the conversion from red bone marrow to yellow bone marrow begins in the peripheral skeleton 
and then propagates to the axial skeleton. The differences between the fat fractions computed in the current study and in the studies of Schraml et al. and Bolan et al. can be explained by the differences in age [19-23] and health status [4,5] of the populations enrolled in these studies. An additional heterogeneity of bone marrow T1 and T2* relaxation times across sites was also observed in the current study, with variations of the T1 of water linked to the variations of the fat fraction, and $T 2^{*}$ of fat significantly longer than $T 2^{*}$ of water in sites where fat fractions were higher than $50 \%$.

In the current study, a strong negative correlation between the T1 of water and the fat fraction was computed in bone marrow sites with high fat fractions (epiphyses of the humerus and the femur). Such a correlation has already been reported in vitro by Hu et al. [28] who measured with spectroscopy the T1 of water in oil-water emulsions with increasing fat fractions. They observed that the $\mathrm{T} 1$ of water attained values close to the $\mathrm{T} 1 \mathrm{of}$ fat in emulsions with high fat fractions, and conversely that the T1 of fat attained values close to the T1 of water in emulsions with low fat fractions. Hu et al. explained this change of water T1 by the modifications of the molecular lattice that occur when fat predominates: the micro environmental changes induced by the presence of fat molecules shorten the T1 relaxation time of water molecules. These interactions are not as straightforward for lattices with intermediate fat fractions where water and fat molecules form a complex micro environment [28]. The same phenomenon was observed here in vivo. The inter-individual variability of the T1 of water found in the current study could thus be explained by the inter-individual variability of the fat fractions.

There are complex interactions governing the value of $\mathrm{T}^{*}$ in bone marrow. On one hand, the susceptibility differences between lipid and water phases increase local inhomogeneities in the magnetic field and accelerate the transverse relaxation of water protons [29]. This phenomenon shortens the T2* of water with increasing fat fractions. Conversely, the increase of the fat fraction makes the lipid phase more homogeneous and increases the $\mathrm{T} 2$ * of fat. The value of T2* also depends of the microarchitecture of the trabecular bone surrounding the bone marrow $[8,10,11]$. Mechanical studies on the structure of the trabecular 
bone have shown the presence of regional variations in the volume and the porosity of the trabecular bone $[30,31]$. For instance, the femur has a higher volume of trabecular bone and smaller pores than the vertebral body [30]. The mesh of trabecular bone creates local inhomogeneities in the magnetic field that shorten the $\mathrm{T}^{*}$ of the surrounding tissues [16]; regional variations in the volume of trabecular bone can thus influence the value of T2*. All these parameters influence the values of $T 2^{*} \mathrm{~W}$ and $\mathrm{T} 2^{*} \mathrm{f}$ and make it difficult to interpret. When considering all the parameters measured in the five sites of bone marrow studied, the most reproducible site for measurements of bone marrow fat fraction, $\mathrm{T} 1$ and $\mathrm{T} 2{ }^{*}$ relaxation times across volunteers was the ilium, which is the most common site for bone marrow biopsy.

With respect to the choice of the number of echoes employed in the current study, only few studies in the literature modelled a dual-T2* decay in the signal equation [17,34]. These studies used a number of echoes comprised between 4 [17] and 6 [34]. Thus, our choice of number of echoes is in line with the previous studies on dual-T2* modelling.

The current study has some limitations. Given the multiparametric nature of the model, data fitting was performed on ROIs and not on a voxel-basis to increase the stability of the fit. This prevented us from generating maps of the computed parameters. Furthermore, errors in the measurements of the parameters, especially $\mathrm{T} 1$, could arise from B1 inhomogeneities as shown by Tsai et al. in [35]. This limitation could be overcome by acquiring a 3D B1 maps and correcting the T1 for B1. Another limitation of our study is the small number of volunteers that did not allow us to identify age- or sex-specific properties of bone marrow across sites, and no measurements were performed on patients.

In conclusion, we have shown the feasibility of measuring the fat fraction, $\mathrm{T} 1$ and $\mathrm{T} 2{ }^{*}$ relaxation times of water and fat in bone marrow in multiple locations with a fast chemicalshift-encoded 3D multi-gradient-echo sequence. Regional variations were computed, and a strong correlation between the fat fraction and the $\mathrm{T} 1$ of water in bones with high fat fractions was found. 


\section{Acknowledgments}

The authors would like to thank Professor Jean-Christophe Ferré and the radiographers of Rennes University Hospital for their kind support.

\section{References}

[1] Snyder, W.S., Cook, M.J., Nasset, E.S., Karhausen, L.R., Parry Howells, G., and Tipton, I.H. Anatomical values for reference man. In: The International Commission on Radiological Protection. Report of the task group on reference man, 1st edition. Pergamon Press: Oxford, $1975 ; 96-7$

[2] Travlos, G.S. Normal structure, function, and histology of the bone marrow. Toxicol pathol. 2006; 34: 548-565.

[3] Schraml, C., Schmid, M., Gatidis, S., Schmidt, H., Fougère, C., Nikolaou, K., et al. Multiparametric analysis of bone marrow in cancer patients using simultaneous PET/MR imaging: correlation of fat fraction, diffusivity, metabolic activity, and anthropometric data. $J$ Magn Reson Imaging. 2015; 42: 1048-1056.

[4] Takasu, M., Tani, C., Sakoda, Y., Ishikawa, M., Tanitame, K., Date, S., et al. Iterative decomposition of water and fat with echo asymmetry and least-squares estimation (IDEAL) imaging of multiple myeloma: initial clinical efficiency results. Eur Radiol. 2012; 22: 11141121.

[5] Bolan, P.J., Arentsen, L., Sueblinvong, T., Zhang, Y., Moeller, S., Carter, J.S., et al. Water-fat MRI for assessing changes in bone marrow composition due to radiation and chemotherapy in gynecologic cancer patients. J Magn Reson Imaging. 2013; 38: 1578-1584. [6] Yeung, D.K.W., Griffith, J.F., Antonio, G.E., Lee, F.K.H., Woo, J., and Leung, P.C. Osteoporosis is associated with increased marrow fat content and decreased marrow fat unsaturation: a proton MR spectroscopy study. J Magn Reson Imaging. 2005; 22: 279-285. [7] Li, X., Kuo, D., Schafer, A.L., Porzig, A., Link, T.M., Black, D., et al. Quantification of vertebral bone marrow fat content using 3 Tesla MR spectroscopy: reproducibility, vertebral variation, and applications in osteoporosis. J Magn Reson Imaging. 2011; 33: 974-9. 
[8] Kühn, J.P., Hernando, D., Meffert, P.J., Reeder, S., Hosten, N., Laqua, R., et al. Protondensity fat fraction and simultaneous $\mathrm{R}^{*}$ estimation as an MRI tool for assessment of osteoporosis. Eur Radiol. 2013; 23: 3432-9.

[9] Funke, M., Bruhn, H., Vosshenrich, R., Rudolph, O., and Grabbe, E. The determination of the T2* relaxation time for characterizing trabecular bone. RoFo Fortschr Geb Rontgenstr Neuen Bildgeb Verfahr. 1994; 161: 58-63.

[10] Wehrli, F.W., Ford, J.C., and Haddad, J.G. Osteoporosis: clinical assessment with quantitative MR imaging in diagnosis. Radiology. 1995; 196: 631-641.

[11] Maris, T.G., Damilakis, J., Sideri, L., Deimling, M., Papadokostakis, G., Papakonstantinou, O., et al. Assessment of the skeletal status by MR relaxometry techniques of the lumbar spine: comparison with dual X-ray absorptiometry. Eur J Radiol. 2004; 50: 245-256.

[12] Biffar, A., Baur-Melnyk, A., Schmidt, G.P., Reiser, M.F., and Dietrich, O. Multiparameter MRI assessment of normal-appearing and diseased vertebral bone marrow. Eur Radiol. 2010; 20: 2679-2689.

[13] Träber, F., Block, W., Layer, G., Bräucker, G., Gieseke, J., Kretzer, S., et al. Determination of $\mathrm{H}$ relaxation times of water in human bone marrow by fat-suppressed turbo spin echo in comparison to MR spectroscopic methods. J Magn Reson Imaging. 1996; 6: $541-8$.

[14] Le Ster, C., Gambarota, G., Lasbleiz, J., Guillin, R., Decaux, O., and Saint-Jalmes, H. Breath-hold MR measurements of fat fraction, $\mathrm{T} 1$, and $\mathrm{T} 2{ }^{*}$ of water and fat in vertebral bone marrow. J Magn Reson Imaging. 2016; 44: 549-555.

[15] Zhong, X., Nickel, M.D., Kannengiesser, S.A., Dale, B.M., Kiefer, B., and Bashir, M.R. Liver fat quantification using a multi-step adaptive fitting approach with multi-echo GRE imaging. Magn Reson Med. 2014; 72: 1353-1365.

[16] Karampinos, D. C., Melkus, G., Baum, T., Bauer, J. S., Rummeny, E. J., and Krug, R. Bone marrow fat quantification in the presence of trabecular bone: Initial comparison 
between water-fat imaging and single-voxel MRS. Magn Reson Med. 2014; 71: 1158-1165.

[17] Bydder, M., Yokoo, T., Hamilton, G., Middleton, M.S., Chavez, A.D., Schwimmer, J.B., et al. Relaxation effects in the quantification of fat using gradient echo imaging. Magn Reson Imaging. 2008; 26: 347-359.

[18] Schick, F. Bone marrow NMR in vivo. Progress in Nuclear Magnetic Resonance Spectroscopy. 1996; 29: 169-227.

[19] Kugel, H., Jung, C., Schulte, O., and Heindel, W. Age-and sex-specific differences in the 1H-spectrum of vertebral bone marrow. J Magn Reson Imaging. 2001; 13: 263-8. [20] Griffith, J.F., Yeung, D.K.W., Ma, H.T., Leung, J.C.S., Kwok, T.C.Y., and Leung, P.C. Bone marrow fat content in the elderly: a reversal of sex difference seen in younger subjects. J Magn Reson Imaging. 2012; 36: 225-230.

[21] Schellinger, D., Lin, C.S., Fertikh, D., Lee, J.S., Lauerman, W.C., Henderson, F., et al. Normal Lumbar Vertebrae: Anatomic, Age, and Sex Variance in Subjects at Proton MR Spectroscopy_Initial Experience 1. Radiology. 2000; 215:910-6.

[22] Liney, G.P., Bernard, C.P., Manton, D.J., Turnbull, L.W., and Langton, C.M. Age, gender, and skeletal variation in bone marrow composition: a preliminary study at 3.0 Tesla. J Magn Reson Imaging. 2007; 26: 787-793.

[23] Dieckmeyer, M., Ruschke, S., Cordes, C., Yap, S.P., Kooijman, H., Hauner, H., et al. The need for T2 correction on MRS-based vertebral bone marrow fat quantification: implications for bone marrow fat fraction age dependence. NMR Biomed. 2015; 28: 432-9. [24] Baum, T., Yap, S.P., Dieckmeyer, M., Ruschke, S., Eggers, H., Kooijman, H., et al. Assessment of whole spine vertebral bone marrow fat using chemical shift-encoding based water-fat MRI. J Magn Reson Imaging. 2015; 42: 1018-1023.

[25] Schick, F., Bongers, H., Jung, W.I., Eismann, B., Skalej, M., Einsele, H., et al. Proton relaxation times in human red bone marrow by volume selective magnetic resonance spectroscopy. Applied Magnetic Resonance. 1992; 3: 947-963. 
[26] Berglund, J., Johansson, L., Ahlström, H., and Kullberg, J. Three-point dixon method enables whole-body water and fat imaging of obese subjects. Magn Reson Med. 2010; 63: 1659-1668.

[27] Cristy, M. Active bone marrow distribution as a function of age in humans. Phys Med Biol. 1981; 26: 389-400.

[28] Hu, H.H., and Nayak, K.S. Change in the proton T1 of fat and water in mixture. Magn Reson Med. 2010; 63: 494-501.

[29] Mulkern, R.V., Hung, Y.P., Ababneh, Z., Maier, S.E., Packard, A.B., Uluer, M.C., et al. On the strong field dependence and nonlinear response to gadolinium contrast agent of proton transverse relaxation rates in dairy cream. Magn Reson Imaging. 2005; 23: 757-764. [30] Amling, M., Herden, S., Pösl, M., Hahn, M., Ritzel, H., and Delling, G. Heterogeneity of the skeleton: comparison of the trabecular microarchitecture of the spine, the iliac crest, the femur and the calcaneus. J Bone Miner Res. 1996; 11: 36-45.

[31] Eckstein, F., Matsuura, M., Kuhn, V., Priemel, M., Müller, R., Link, T.M., et al. Sex Differences of Human Trabecular Bone Microstructure in Aging Are Site-Dependent. J Bone Miner Res. 2007; 22: 817-824.

[32] Liu, C.Y., McKenzie, C.A., Yu, H., Brittain, J.H., and Reeder, S.B. Fat quantification with IDEAL gradient echo imaging: correction of bias from T1 and noise. Magn Reson Med. 2007; 58: 354-364.

[33] Karampinos, D.C., Ruschke, S., Dieckmeyer, M., Eggers, H., Kooijman, H., Rummeny, E.J., et al. Modeling of T2* decay in vertebral bone marrow fat quantification. NMR Biomed. 2015; 28: 1535-1542.

[34] Chebrolu, V. V., Hines, C. D., Yu, H., Pineda, A. R., Shimakawa, A., McKenzie, C. A., et al. Independent estimation of $T^{*} 2$ for water and fat for improved accuracy of fat quantification. Magn Reson Med. 2010;63: 849-857.

[35] Tsai, W. C., Kao, K. J., Chang, K. M., Hung, C. F., Yang, Q., Lin, C. Y. E., and Chen, C. M. B1 field correction of T1 estimation should be considered for breast dynamic contrastenhanced MR imaging even at 1.5 T. Radiology. 2016;282: 55-62. 


\section{TABLE/FIGURE LEGENDS}

Table 1. FF, $\mathrm{T} 1$ and $\mathrm{T}^{*}$ relaxation times of water and fat in healthy volunteers. Across-ROI mean values of fat fraction, $\mathrm{T} 1$ and $\mathrm{T} 2^{*}$ relaxation times of water and fat computed in the bone marrow of the humerus (2 ROIs), sternum (1 ROI), lumbar vertebrae (5 ROls), ilium (2 ROIs) and femur (2 ROIs) in healthy volunteers. The coefficients of variation are indicated in parentheses. The coefficients of determination $\left(R^{2}\right)$ are given in the last line of the table.

Table 2. t-test between water and fat parameters for $\mathrm{T} 1$ and $\mathrm{T} 2^{*}$ relaxation times in the five sites of bone marrow studied. The differences between T1w and T1f were significant in all bone marrow sites and the differences between $T 2^{*} \mathrm{~W}$ and $T 2^{*} \mathrm{f}$ were significant in the humerus, ilium and femur.

Figure 1. MR images of a 25-year-old woman acquired at the first echo (TE=1.18ms) with a flip angle of $5^{\circ}$. From the left to the right, the bone marrow sites correspond to the sternum, humerus, lumbar spine, ilium and femur, respectively. The regions of interest used to sample the signal are displayed in each site.

Figure 2. Example of two datasets and their corresponding fittings for the acquisitions with the flip angles $5^{\circ}$ (red) and $15^{\circ}$ (blue) in the L5 vertebra (left) and in the left humerus (right) of a volunteer. The algorithm can adapt to a large range of oscillation width in combination with $\mathrm{T}^{*}$ decrease and provides fits of good quality with, for instance, a fat fraction of $46 \%$ in the L5 vertebra (left panel) and $86 \%$ in the humerus (right panel).

Figure 3. Scatter plot of bone marrow fat fraction versus T1w in the humerus (blue circles) and the femur (black stars). A high negative correlation was measured between these parameters as represented by the continuous line (humerus) and dashed line (femur) 

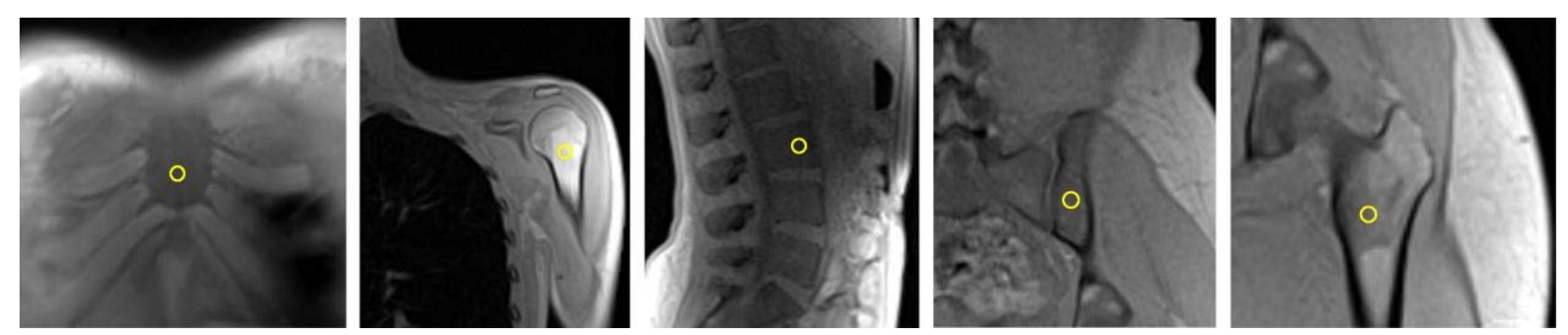

Figure 1

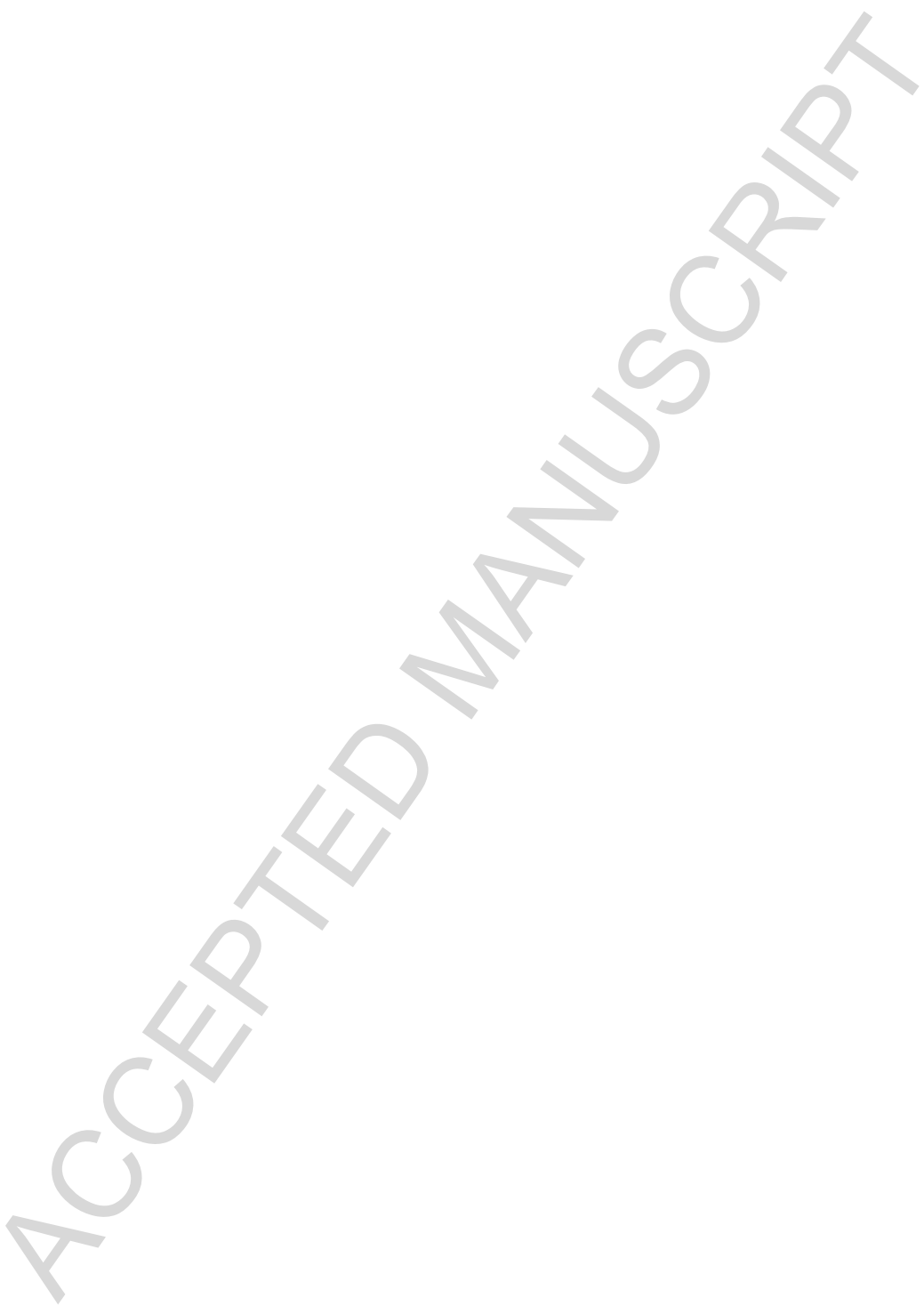



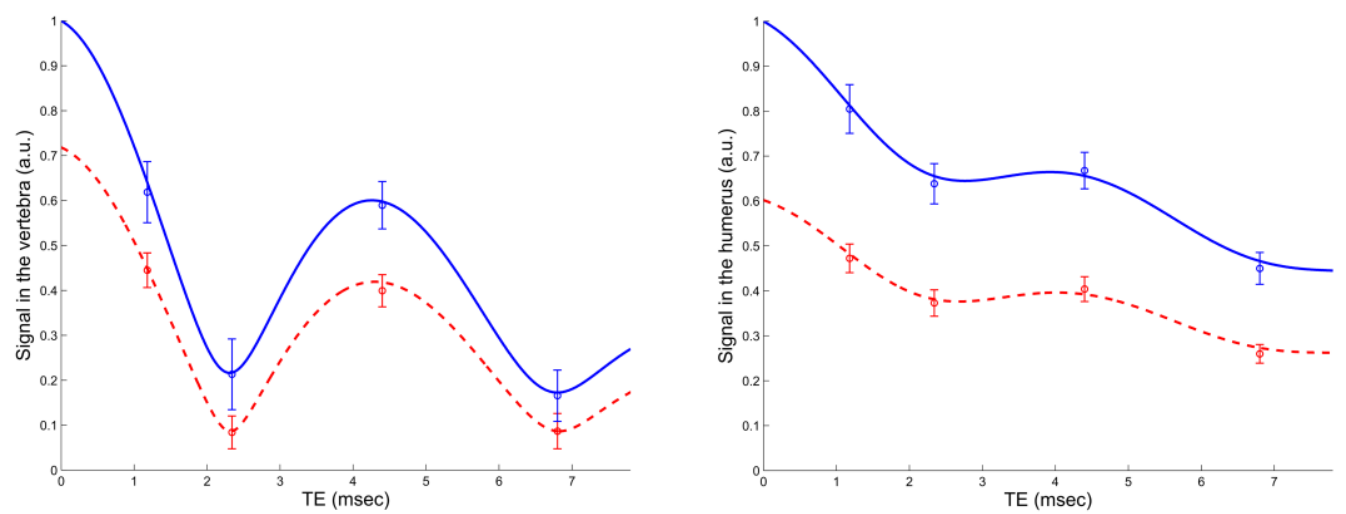

Figure 2 


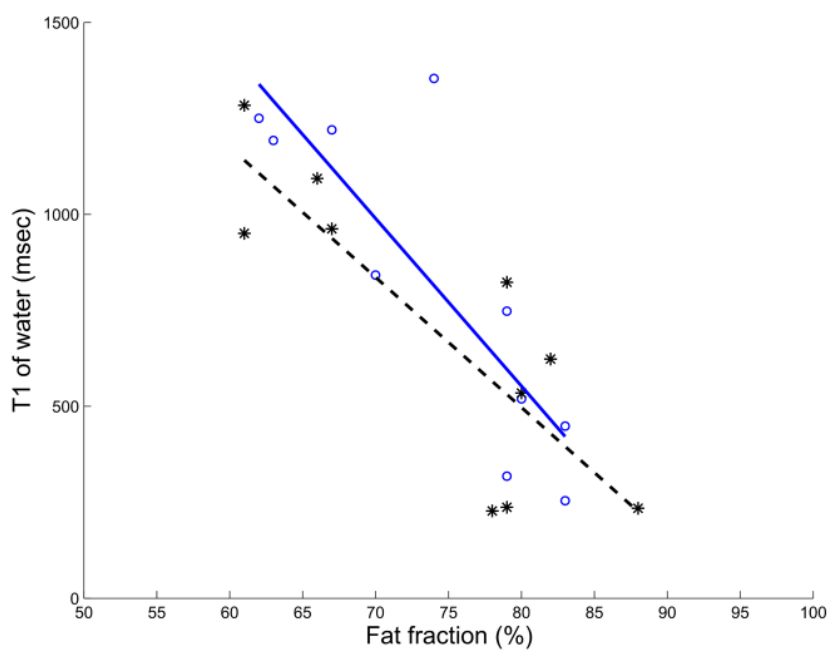

Figure 3 
Table 1. FF, $\mathrm{T} 1$ and $\mathrm{T}^{*}$ relaxation times of water and fat in healthy volunteers. Across-ROI mean values of fat fraction, $\mathrm{T} 1$ and $\mathrm{T} 2^{*}$ relaxation times of water and fat computed in the bone marrow of the humerus ( $2 \mathrm{ROIs})$, sternum ( $1 \mathrm{ROI}$ ), lumbar vertebrae ( $5 \mathrm{ROIs}$ ), ilium (2 ROIs) and femur (2 ROIs) in healthy volunteers. The coefficients of variation are indicated in parentheses. The coefficients of determination $\left(R^{2}\right)$ are given in the last line of the table.

\begin{tabular}{cccccc}
\hline & Humerus & Sternum & Vertebra & llium & Femur \\
\hline FF (\%) & $74(11 \%)$ & $45(32 \%)$ & $42(15 \%)$ & $50(8 \%)$ & $74(13 \%)$ \\
T1w (msec) & $815(41 \%)$ & $706(40 \%)$ & $621(18 \%)$ & $771(37 \%)$ & $697(54 \%)$ \\
T1f (msec) & $279(12 \%)$ & $224(41 \%)$ & $330(6 \%)$ & $283(12 \%)$ & $256(9 \%)$ \\
T2*w (msec) & $4.7(75 \%)$ & $11.4(34 \%)$ & $12.7(42 \%)$ & $8.6(21 \%)$ & $5.2(55 \%)$ \\
T2*f (msec) & $30.0(8 \%)$ & $27.7(21 \%)$ & $15.9(24 \%)$ & $23.5(8 \%)$ & $23.1(16 \%)$ \\
$\mathrm{R}^{2}$ & 0.9998 & 0.9993 & 0.9993 & 0.9994 & 0.9999 \\
\hline
\end{tabular}


Table 2. t-test between water and fat parameters for $\mathrm{T} 1$ and $\mathrm{T} 2^{*}$ relaxation times in the five sites of bone marrow studied. The differences between T1w and T1f were significant in all bone marrow sites and the differences between $T 2^{*} \mathrm{~W}$ and $\mathrm{T} 2^{*} \mathrm{f}$ were significant in the humerus, sternum, ilium and femur.

\begin{tabular}{cccccc}
\hline & Humerus & Sternum & Vertebra & llium & Femur \\
\hline p-value T1 & $<0.001$ & 0.007 & $<0.001$ & $<0.001$ & 0.002 \\
p-value T2* & $<0.001$ & $<0.001$ & 0.04 & $<0.001$ & $<0.001$ \\
\hline
\end{tabular}

